\title{
Diagnostic Accuracy of Gene Xpert on Bronchoscopy Washings in Patients with Suspected Pulmonary Tuberculosis
}

\author{
Waseem A. Khan, ${ }^{1}$ Nayyar Manzoor, ${ }^{2}$ Asad Javaid, ${ }^{3}$ M. Saqib Musharaf, ${ }^{4}$ Nausheen Sohail, ${ }^{5}$ \\ Nighat Majeed $^{6}$
}

\begin{abstract}
Objective: The objective of this study was to determine the diagnostic accuracy of GeneXpert assay of bronchial washing in detecting pulmonary tuberculosis in suspected TB patients with no sputum production taking culture of bronchial washing as gold standard.

Methods: This is cross sectional study. Data was collected through structured questionnaire. Patients had bronchoscopy according to standard protocol. Their bronchial washings were sent for culture and GeneXpert assay for Mycobacterium Tuberculosis. Data analysis was done using SPSS 20 version.

Results: From one hundred and seventy patients the age was observed as 16-60 years. AFB was detected by GeneXpert assay in $45.3 \%$ patients. AFB was detected by culture report in $47.6 \%$ patients. The sensitivity of GeneXpert was observed as $87.65 \%$, specificity was $93.26 \%$, and diagnostic accuracy was calculated as $90.59 \%$. By using chi-square t-test, there was significant association found between GeneXpert and Culture report in both gender in all age groups with any duration of symptoms with $p$-value $=0.000$.

Conclusion: The GeneXpert assay was highly effective for detecting pulmonary tuberculosis in suspected TB patients who do not have sputum production.

Key Words: GeneXpert Assay, Culture Report, Bronchial Washing, Pulmonary Tuberculosis

How to Cite: Waseem A. K, Manzoor N, Javaid A, Musharaf M.S, Sohail N, Majeed N. Diagnostic accuracy of gene xpert on bronchoscopy washings in patients with suspected pulmonary tuberculosis. Esculapio.2020;16(04):33-36.

DOI: https://doi.org/10.51273/esc20.251647
\end{abstract}

\section{Introduction}

$\mathrm{T}$ uberculosis is caused by Mycobacterium speThe most common species is Mycobacte- rium Tuberculosis which usually infects humans in most cases. But other species can also infect humans like Mycobacterium bovis, Mycobacterium africa- num,

Mycobacterium microti and Mycobacterium canettii. ${ }^{1}$ Tuberculosis is an air born infection and infectious droplets remain in environment when infected person speaks, sneezes and coughs. ${ }^{1}$ All individuals who are exposed do not get infection. Whether the infection will spread to other individual

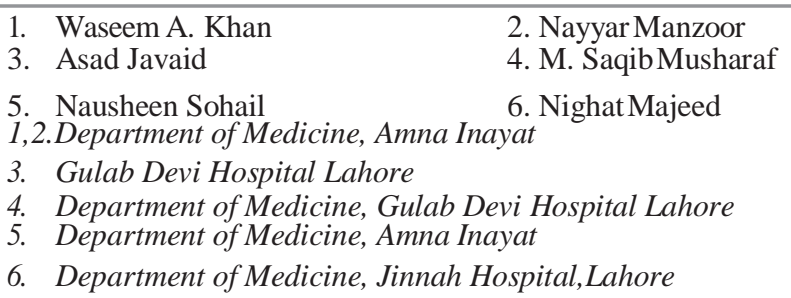

\section{Correspondence:}

Dr. Waseem A. Khan, Department of Medicine, Amna Inayat E-mail: drwaseem77@gmail.com

$\begin{array}{ll}\text { Submission Date: } & 12-11-2020 \\ \text { 1st Revision Date: } & 14-11-2020 \\ \text { Acceptance Date: } & 19-11-2020\end{array}$

depend on certain factors like immunity of individual, duration of exposure and infectiveness of the source. ${ }^{1}$ It is the immunity of the individual which contain the infection and does not allow its spread. In about $5 \%$ individual infection is not controlled and individual develops active TB within 1-2 years. ${ }^{2}$ This is called primary tuberculosis. In other $5 \%$ individuals the immune system contain infection to localized site but mycobacterium remains in dormant stage and reactivate later on when the immunity of individual becomes weak. ${ }^{2}$ This category is named as postprimary or reactivation of tuberculosis. The remaining $90 \%$ of the individuals will harbor mycobacterium in tissue but will be asymptomatic and this category is called as latent tuberculosis. Tuberculosis infects one third of world population ${ }^{3}$ and causes significant morbidity and mortality. It is major public health problem because it spreads through droplet infection. ${ }^{3}$ Milllions of people get infected and a million people die each year because of this infectious disease. ${ }^{1}$ Pakistan belongs to the countries which has highest burden of the disease in the world. Pakistan is 4th among the twelve countries with high number of 
missing TB cases. ${ }^{6}$ Major diagnostic procedures employed for diagnosing pulmonary tuberculosis are sputum smear, culture for detection of Acid-Fast bacilli (AFB) and chest radiography. In those patients who are highly suspected of tuberculosis based on history and radiology it is pertinent to demonstrate presence of AFB through sputum smear to avoid unnecessary empiric therapy.

Active disease may manifest with minimal symptoms initially but as the disease progresses over months then overt symptoms develop. ${ }^{4}$ Active tuberculosis manifests with symptoms of fever, productive cough, night sweats weight loss and fatigue. Sometimes patient may present with hemoptysis with typical symptoms. ${ }^{4}$ The clinician should have high index of suspicion since the disease has insidious onset and nonspecific symptoms.

Extrapulmonary tuberculosis results from hematogenous spreads to different organs of body like pleura, lymph nodes and larynx. Most extrapulmonary disease is not contagious, but laryngeal tuberculosis is exception. Chest $\mathrm{x}$-ray may be normal in cases of extrapulmonary TB. Miliary tuberculosis results from hematogenous route and can cause numerous tiny lesions throughout the lung field measuring 1$3 \mathrm{~mm}$ and it can disseminate to other organs of body like central nervous system, eye and liver.

In postprimary $\mathrm{TB}$ patient, upper zones are involved usually. ${ }^{5,7}$ The radiological features of postprimary TB includes patchy consolidation, dense consolidaion and cavitation involving apical and posterior segments of upper lobes and superior segments of lower lobes. ${ }^{5}$ In $5 \%$ of postprimary TB patients only lung bases are involved. ${ }^{2}$ Cavitation is common finding seen in $20 \%-45 \%$ cases. CT scan chest may be used when chest $\mathrm{x}$-ray is not diagnostic but $\mathrm{CT}$ is not routinely required for diagnosing TB. ${ }^{8}$

Not all patients expectorate adequate sputum and it's minimal in amount some time that cannot be collected. In those cases where there is radiological evidence of lesion but no sputum production making diagnosis is a challenge. Patients who have smear negative tuberculosis they are less infectious than sputum smear positive patients. ${ }^{9,10}$ However patients who are smear negative but culture positive tuberculosis they can spread mycobacterium tuberculosis. ${ }^{112}$ In those cases where there is scanty sputum or no sputum production bronchoscopy can be helpful in making diagnosis. Bronchial washings can be taken and GeneXpert and culture can be done on sample. In previous study GeneXpert was done on bronchial washings and showed encouraging results. Out of 120 patients, 83(69.2\%) patients were detected having active pulmonary tuberculosis on bronchial washings in sputum scarce TB patients. ${ }^{13}$

\section{Methods}

This crossectional study was conducted in the department of pulmonology of Jinnah Hospital lahore. Suspected pulmonary tuberculosis patient of either sex having ages 16 to 60 years with fever and cough for more than 6 months but no sputum production were enrolled after approval from ethical committee of research at Jinnah Hospital. Patients who were diagnosed of pulmonary TB and received anti-tuberculosis medication for 2 weeks and those patients who fall in the definition of category-2 patients were excluded. Informed consent was taken and data was collected through questionnaire. Patients had bronchoscopy procedure according to the protocol. Their bronchial washings were sent for culture for Mycobacterium Tuberculosis, and GeneXpert assay for presence of AFB immediately to pathology laboratory of Allama Iqbal Medical College. Results of both GeneXpert assay and culture were collected. Pulmonary tuberculosis was diagnosed by both GeneXpert and positive culture for Mycobacterium Tuberculosis. Sensitivity, specificity and diagnostic accuracy of GeneXpert assay was calculated using culture as gold standard. Data analysis was done by using SPSS version 20 .

\section{Results}

From 170 patients the minimum age was observed as 16 years and maximum age was 60 years with Mean \pm $\mathrm{SD}$ as $39.08 \pm 13.74$ years. The minimum duration of symptoms was observed as 6 months and maximum duration of symptoms was 18 months with Mean \pm SD as $11.32 \pm 3.24$ months. AFB was detected by GeneXpert assay in 77(45.3\%) and by culture report in $81(47.6 \%)$ patients. The sen-sitivity of GeneXpert was observed as $87.65 \%$, specificity was $93.26 \%$, positive predicted value was $92.21 \%$, negative predicted value was $89.25 \%$ and diagnostic accuracy was calculated as $90.59 \%$. By using chi-square t-test, there was significant asso-ciation found between GeneXpert and Culture report in less than 40 years of age group with $p$-value $=0.000$. In more than 40 years 
of age group, there was significant association found between GeneXpert and Culture report with p-value $=0.000$. There was significant association found between GeneXpert and Culture report in male with p-value $=0.000$. In female, there was significant association found between GeneXpert and Culture report with $p$-value $=0.000$. There was significant association found between GeneXpert and Culture report in less than one year duration of symptoms group with $\mathrm{p}$-value $=0.000$. In more than one year duration of symptoms group, there was significant association found between GeneXpert and Culture Table 1: Diagnostic Accuracy of GeneXpert on Bronchoscopy Washings

\begin{tabular}{lccc}
\multirow{2}{*}{ GeneXpert } & \multicolumn{2}{c}{ Culture Report } & Total \\
\cline { 2 - 3 } & AFB Detected AFB not Detected & \\
\hline AFB detected & $\mathrm{TP}=71$ & $\mathrm{FP}=6$ & 77 \\
AFB not detected & $\mathrm{FN}=10$ & $\mathrm{TN}=83$ & 93 \\
Total & 81 & 89 & 170 \\
\hline
\end{tabular}

Table 2: Stratification with respect to Age $(n=170)$

\begin{tabular}{|c|c|c|c|c|c|}
\hline \multirow[b]{2}{*}{ Age } & \multirow[b]{2}{*}{ GeneXpert } & \multicolumn{2}{|c|}{ Culture Report } & \multirow{2}{*}{ Total } & \multirow{2}{*}{$\begin{array}{c}\text { P- } \\
\text { value }\end{array}$} \\
\hline & & $\begin{array}{c}\text { AFB } \\
\text { Detected }\end{array}$ & $\begin{array}{l}\text { AFB not } \\
\text { Detected }\end{array}$ & & \\
\hline$<40$ & AFB detected & 27 & 3 & 30 & 0.000 \\
\hline \multirow[t]{2}{*}{ year } & AFB not detected & 10 & 35 & 45 & \\
\hline & Total & 37 & 38 & 75 & \\
\hline$\geq 40$ & AFB detected & 44 & 3 & 47 & 0.000 \\
\hline \multirow[t]{2}{*}{ year } & AFB not detected & 0 & 48 & 48 & \\
\hline & Total & 44 & 51 & 95 & \\
\hline
\end{tabular}

Table 3: 3 Stratification with respect to Duration of Symptoms $(n=170)$

\begin{tabular}{|c|c|c|c|c|c|}
\hline \multirow{2}{*}{$\begin{array}{c}\text { Duration } \\
\text { of } \\
\text { Symptoms }\end{array}$} & \multirow[b]{2}{*}{ GeneXpert } & \multicolumn{2}{|c|}{ Culture Report } & \multirow[b]{2}{*}{ 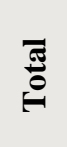 } & \multirow{2}{*}{$\begin{array}{c}\text { P- } \\
\text { value }\end{array}$} \\
\hline & & $\begin{array}{c}\text { AFB } \\
\text { Detected }\end{array}$ & $\begin{array}{l}\text { AFB not } \\
\text { Detected }\end{array}$ & & \\
\hline \multirow[t]{3}{*}{$<1$ year } & AFB detected & 34 & 3 & 37 & 0.000 \\
\hline & AFB not detected & 5 & 41 & 46 & \\
\hline & Total & 39 & 44 & 83 & \\
\hline \multirow[t]{3}{*}{$\geq 1$ year } & AFB detected & 37 & 3 & $40 c$ & 0.000 \\
\hline & AFB not detected & 5 & 42 & 47 & \\
\hline & Total & 42 & 45 & 87 & \\
\hline
\end{tabular}

report with p-value $=0.000$.

\section{Discussion}

The objective of this research is to determine the diagnostic efficacy of GeneXpert assay of bronchial washing in detecting pulmonary tuberculosis in suspected patients with no sputum production. In our study there was significant association found between GeneXpert and Culture report in both gender in all age groups with any duration of symptoms with pvalue $=0.000$. Mostly pulmonary tuberculosis is diagnosed on clini-cal grounds and with radiology in patients when there is typical history. The diagnostic accuracy of different modalites have been studied before like clinical assessment, radiology and sputum microscopy. In previous study, out of 364 TB patients, $35.1 \%$ were diagnosed on clinical grounds and radiology, $20.8 \%$ on radiological, and $17.58 \%$ on the basis of clinical evaluation. Sputum smear examination for Mycobac-terium tuberculosis was done in $85.6 \%$ patients, of these $31.9 \%$ were positive. ${ }^{14}$ Previous studies have evaluated the efficacy of GeneXpert assay on pulmonary and extrapulmonary samples and results are very encouraging. For pulmo-nary samples the sensitvity and specificity were $90.6 \%$ and $94.3 \%$. For extrapulmonary samples they were $100 \%$ and $91.6 \%$. For sputum smear negative sam-ples the sensitvity, specificity, positive predictive value and negative predictive values were $86.3 \%, 93 \%, 79 \%$, and $95.6 \% .^{15}$ Our study has comparable results with previous study. In our study The sensi-tivity of GeneXpert was observed as $87.65 \%$, speci-ficity was $93.26 \%$, positive predicted value was $92.21 \%$, negative predicted value was $89.25 \%$.

In cases where there is no sputum production but high suspicion of tuberculosis, sputum induction and bronchoscopy can be done to collect sample. Bronchoscopy is accepted modality for diagnosis when saline induction is not helpful. ${ }^{16}$ In one study where sputum was not produced but x-ray was showing active disease, flexible fiberoptic bronchoscope was used in making diagnosis. In that study, two hundred and seventy five suspected TB patients based on chest radiology, fiberoptic bronchoscopy was done and bronchial brushings and transbronchial biopsy samples colllected. $89(32.4 \%)$ were diagnosed as active pulmonary TB. In $60(67.4 \%)$ of these patients the diagnosis was made by bronchoscopy. ${ }^{17}$ There are very few studies who have used bronchial washings and GeneXpert and using culture as gold standard in sputum scarce TB patients. In previous local study bronchoscopy was done to collect bronchial washing sample and GeneXpert done. ${ }^{13}$ Out of 120 patients eighty three patients were diagnosed as active pulmonary TB by this approach. Our study is unique in a way that it not only determines the role of GeneXpert 
on bronchial washing sample but also determine the efficacy of culture as well on same patient.

\section{Conclusion}

The GeneXpert assay has high diagnostic accuracy for detecting pulmonary $\mathrm{TB}$ in suspected patients where $\mathrm{x}$-ray is suggestive of active disease but there is no sputum production.

\section{Conflict of Interest: $\quad$ None}

\section{References}

1. cdc.gov [internet]. Centers for disease control and prevention, Inc; Self-study modules on tuberculosis. Updated May 11, 2016; Accessed June 14, 2016. Available from: http:// www.cdc.gov/ tb/ education/ ssmodules/.

2. Leung AN. Pulmonary tuberculosis: the essentials. Radiology. 1999Feb;210(2):307-22.

3. Loennroth K, Raviglione M. Global epidemiology of tuberculosis: prospects for control. InSeminars in respiratory and critical care Medicine 2008 Oct (Vol. 29, No.05, pp.481-491).CThieme Medical Publishers.

4. Arango L, Brewin AW, Murray JF. The spectrum of tuberculosis as currently seen in a metropolitan hospital. American Review of Respiratory Disease. 1973 Oct;108(4):805-12.

5. Curvo-Semedo L, Teixeira L, Caseiro-Alves F. Tuberculosis of the chest. European journal of radiology. 2005 Aug 1;55(2):158-72.

6. Baig S, Qayyum S, Saifullah N, Ahmed N. Detection of TB by smear microscopy and GeneXpert MTB/ RIF assay in non expectorating pulmonary TB suspects and pleural TB in high prevalent low income setting. European Respiratory Journal. 2014 Sep 1; 44(Suppl 58).

7. Goodwin RA, Des Prez RM. Apical localization of pulmonary tuberculosis, chronic pulmonary histoplasmosis, and progressive massive fibrosis of the lung. Chest. 1983 May 1;83(5):801-5.

8. Lee SW, Jang YS, Park CM, Kang HY, Koh WJ, Yim JJ, Jeon K. The role of chest CT scanning in TB outbreak investigation. Chest. 2010 May 1;137(5): 1057-64.

9. Tostmann A, Kik SV, Kalisvaart NA, Sebek MM, Verver S, Boeree MJ, van Soolingen D. Tuberculosis transmission by patients with smear-negative pulmo- nary tuberculosis in a large cohort in the Netherlands. Clinical Infectious Diseases. 2008 Nov 1;47(9):1135 $-42$.

10. Tostmann A, Kik SV, Kalisvaart NA, Sebek MM, Verver S, Boeree MJ, van Soolingen D. Tuberculosis transmission by patients with smear-negative pulmonary tuberculosis in a large cohort in the Netherlands. Clinical Infectious Diseases. 2008 Nov 1;47(9): 1135 $-42$.

11. Behr MA, Warren SA, Salamon H, Hopewell PC, De Leon AP, Daley CL, Small PM. Transmission of Mycobacterium tuberculosis from patients smearnegative for acid-fast bacilli. The Lancet. $1999 \mathrm{Feb} 6$; 353(9151):444-9.

12. Hernandez-Garduno E, Cook V, Kunimoto D, Elwood RK, Black WA, FitzGerald JM. Transmission of tuberculosis from smear negative patients: a molecular epidemiology study. Thorax. 2004 Apr 1; 59(4): 286-90.

13. Zuberi FF, Hussain S, Hameed S, Zuberi BF. Role of Bronchial Washing Gene Xpert in Sputum-Scarce Cases of Suspected Pulmonary Tuberculosis. Pakistan Journal of Medical Sciences. 2019 Jan;35(1):211.

14. Khurram M. Pulmonary tuberculosis diagnosis practices. Journal of Rawalpindi Medical College. 2014 Jun 30;18(1):13-5.

15. Ioannidis P, Papaventsis D, Karabela S, Nikolaou S, Panagi M, Raftopoulou E, Konstantinidou E, Marinou I, Kanavaki S. Cepheid GeneXpert MTB/RIF assay for Mycobacterium tuberculosis detection and rifampin resistance identification in patients with substantial clinical indications of tuberculosis and smear-negative microscopy results. Journal of clinical microbiology. 2011 Aug 1;49(8):3068-70.

16. Beatrice M, Nedelcu R, Ulmeanu R, Ciolan G, Tanasescu M, Popescu M, Marica C. Diagnostic role of fiberoptic bronchoscopy in suspected smear negative pulmonary tuberculosis.

17. Willcox PA, Benatar SR, Potgieter PD. Use of the flexible fibreoptic bronchoscope in diagnosis of sputum-negative pulmonary tuberculosis. Thorax. 1982 Aug 1;37(8):598-601.

\section{Author's Contribution}

KAW: Conceived Idea, study draft

JA, MN: Data Collection

MSM: Statistical analysis, interpretation 
